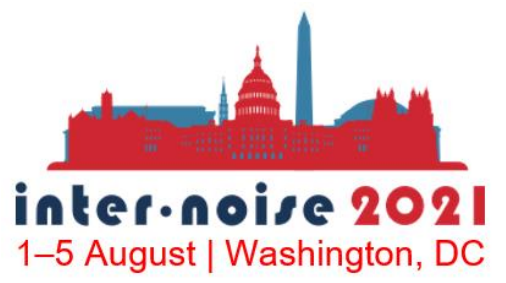

\title{
Monitoring trends in road surface impact on rolling noise emissions
}

\author{
Wout Van Hauwermeiren ${ }^{1}$ \\ Ghent University \\ Technologiepark-Zwijnaarde 126, 9052 - Gent, Belgium \\ Karlo Filipan ${ }^{2}$ \\ ASAsense cvba, Brugge, Belgium \\ Dick Botteldooren ${ }^{3}$ \\ Ghent University \\ Technologiepark-Zwijnaarde 126, 9052 - Gent, Belgium \\ Bert De Coensel ${ }^{4}$ \\ Ghent University \\ Technologiepark-Zwijnaarde 126, 9052 - Gent, Belgium
}

\begin{abstract}
Road surfaces degrade over time due to heavy traffic and weather conditions, which negatively influences both driving comfort and acoustic properties. In addition, the lifetime of a road surface can be increased by performing cost-effective incremental maintenance and this maintenance becomes more expensive when the damages are more severe (cracks, potholes). Current methods such as CPX are performed in a standardized way (using designated equipment and tightly controlled measurement conditions), however budget constraints limit frequent monitoring of surfaces. Therefore, continuous monitoring using ordinary passenger vehicles could be helpful to observe trends in rolling noise emissions and road evenness. Hence, we deployed designated sensor boxes in a number of vehicles that are on the road for other purposes. In addition, advances in calibration of different devices using de-noising autoencoders alleviate the effect of various measurement conditions such as driving speed, braking, accelerating, and temperature. As our innovative methodology has now been on the road for several years, trend analysis becomes possible.
\end{abstract}

\footnotetext{
${ }^{1}$ wout.vanhauwermeiren@ugent.be

${ }^{2}$ karlo.filipan@asasense.com

3 dick.botteldooren@ugent.be

${ }^{4}$ bert.decoensel@ugent.be
} 


\section{INTRODUCTION}

Due to the evolution of road vehicle engine technology and the introduction of hybrid and electric vehicles, the rolling noise component is getting increasingly important in urban sound planning and noise mitigation. The influence of pavement and road surface aging and maintenance have long been recognized as important determinants of rolling noise [1]. In addition, in European cities, stone-based pavements are regularly used partly as a noise based measure to reduce driving speed. Over the last decades CPX (close proximity method) has become the standard method for monitoring noise emission characteristics of road pavements. The method foresees corrections for tire temperature (or air temperature as a proxy) and tire hardness. In [2], Wehr et al. conclude that these corrections can reduce measurement error to $0.3 \mathrm{~dB}$ but also state that some further refinement is needed to measure e.g. the effect of aging. Several CPX trailers are now on the market and round robin tests have been performed [3] showing some significant differences between devices.

The CPX approach starts from the premises of high-end measurement equipment and calibrates and corrects for all known dependencies. Changing the perspective to averaging as a method to reduce the effect of imprecision of the measurement equipment, we proposed an opportunistic [4] approach relying on a large number of vehicles that are on the road for other purposes to monitor road quality and its effect on rolling noise. The measurement done by each vehicle is prone to imprecisions due to type of tire, driving speed, air temperature, external and engine noise. Hence an appropriate on the fly calibration and denoising method is needed to support the effect of averaging and increase convergence rate. In [5] we compared several methods for calibration and denoising and concluded that a denoising autoencoder, a technique based on deep learning, seems most efficient in removing dependence on measurement vehicle and tires as well as dependence on air temperature.

This pavement monitoring concept has now been deployed in several vehicles. This paper focusses on the possible use of the data and the lessons learned.

\section{DATA COLLECTION, CALLIBRATION, AND DENOISING}

\subsection{Data collection}

Sound and vibration data is now being recorded near the rear wheel of over 50 vehicles driving in Flanders. This analysis refers to 33 vehicles that have been on the road for at least 4 months. 1/3 octave bands are calculated 50 times a second from the sound data on the edge device. Statistical properties are calculated once every second from the spectra (minimum, maximum, L10, L90, L50 and Leq). In addition, $\mathrm{x}, \mathrm{y}$, and $\mathrm{z}$ acceleration is sampled at a sample frequency of $100 \mathrm{~Hz}$. 1/3 octave bands are calculated from the $\mathrm{z}$ acceleration in a range of $1 \mathrm{~Hz}$ to $25 \mathrm{~Hz}$. A GPS sensor locates the devices on a $20 \mathrm{~m}$ segment. These data are transmitted to the server together, while the vehicles are driving. The data is kept undisclosed for at least one week for privacy reasons and the trips are truncated to mask origin and destination.

\subsection{Denoising auto-encoder}

The denoising auto-encoder (DAE) is a non-linear deep neural network. DAE encodes input features (statistical noise levels and spectra, acceleration spectra,...) and input conditions (speed, temperature, horizontal acceleration, measurement device) to a bottleneck layers. Target conditions are concatenated to the bottleneck layer: speed, temperature and a device identifier. The result is decoded to an output layer. The output layer consists again of statistical noise levels and spectra. The DAE is trained by pairing up observations made by cars travelling along the same road segment. After training, the model is used by transforming an observation to the whole set of vehicles, as if the observation was made by all these different cars. The average is taken over the device set, so that the output features represents how the car fleet reacts on the road surface. For reference conditions a temperature of $15^{\circ} \mathrm{C}$, target speed at v85 of the road segment and zero horizontal acceleration are used. ${ }^{5}$ Most of the

\footnotetext{
${ }^{5} \mathrm{v} 85$ is the $85^{\text {th }}$ percentile of the speed distribution of a location (15\% of the passages are faster than v85).
} 
observations are made between December 2020 and April 2021. Temperatures with mean $11.8{ }^{\circ} \mathrm{C}$ and standard deviation of $7.4^{\circ} \mathrm{C}$ occurred in the dataset.

To illustrate the effect of the denoising auto-encoder (DAE) on the noise data, the mean of the variance of different measurements within a $20 \mathrm{~m}$ segment, $w$, is compared to the variance of the mean of measurements over multiple segments, $o$, before and after applying the DAE (see [5] for formulas). These variables are calculated for the month March 2021 including around 1.e6 observations and results are shown in Figure 1. As expected the variance over all measurements within a segment is quite high due to differences between cars and tires, driving speed and acceleration/deceleration, temperature, etc. In the frequency region of interest, bringing the roughly $5 \mathrm{~dB}$ variance to an acceptable $0.5 \mathrm{~dB}$ measurement error would require averaging over 100 passages of different vehicles in different conditions in each $20 \mathrm{~m}$ segment. This would be very impractical. However, after applying the DAE, variation within a segment drops to $0.5 \mathrm{~dB}$ in the region of interest - a value not all that different from what has been observed for CPX measurements. Four passages could already bring the standard error on average below $0.25 \mathrm{~dB}$ which is an acceptable level of uncertainty. The variance over segments, $o$, also drops but remains significantly higher than $w$.

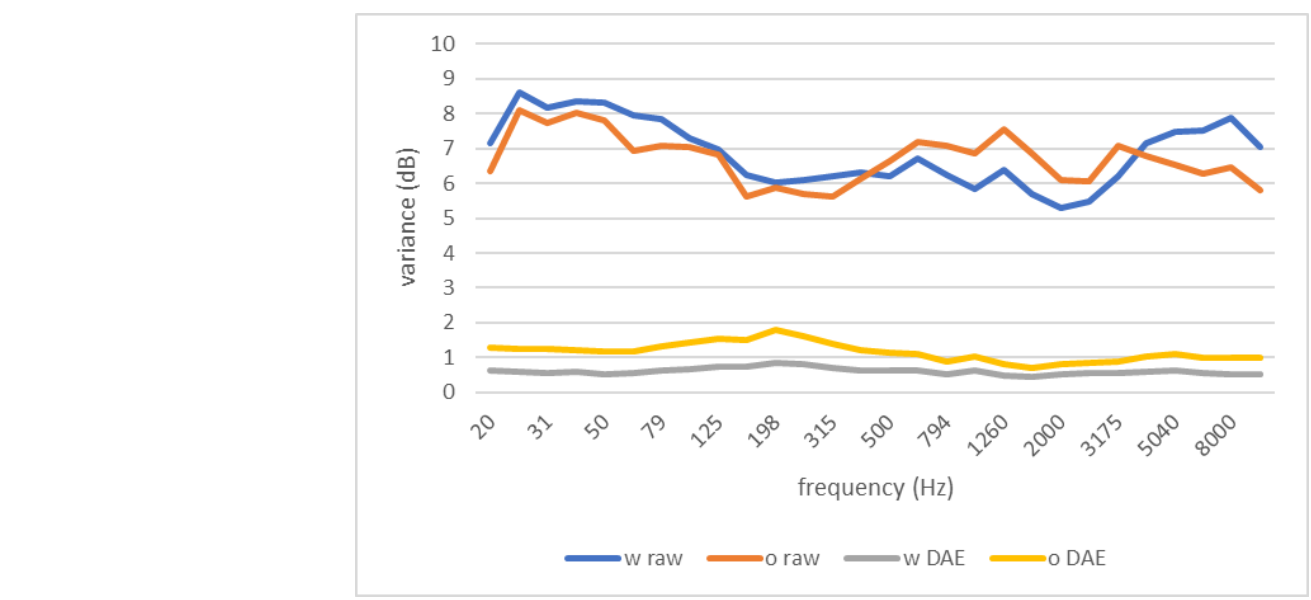

Figure 1 Mean of variance within $20 \mathrm{~m}$ road segments $(w)$ and variance of the mean over $20 \mathrm{~m}$ segments (o) before (raw) and after applying the DAE.

\subsection{Convergence}

In Section 2.2 it was shown that on average the standard error on measurements can be reduced to an acceptable level. However, the opportunistic approach inevitably creates an unbalance between the frequency of use of different routes. Fortunately, the number of passages per time interval will statistically be higher on primary roads than on minor roads and these are precisely the roads one wants to monitor frequently to assess the wear caused by heavy traffic load. Monitoring could be used for assessing the effect of wear on rolling noise and hence a balance needs to be found between frequency of updating the information and the expected noise. This balance is different for primary roads and minor roads and therefore the averaging period should be adapted to the type of road and hence a flexible averaging time window is suggested. The procedure starts from the moment of the last measurements and determines the averaging interval by gradually extending it starting from an initial value of one month to the past until the measurement error in critical frequency bands reaches a predefined level (e.g. 1dB).

\section{RESULTS}

\subsection{Local analysis}

As the opportunistic monitoring system proposed in this paper includes vibrations as well as noise, detailed analysis can reveal not only the effect of the pavement status on the rolling noise, but also underlying defects and structure of the road. In the longer run these could be indicative of emerging cracks, raveling, or potholes that could affect noise emission. Figure 2 shows some examples of combined noise and vibration data over a wide frequency range compared to a one year old SMA on a 
road with speed limit of $90 \mathrm{~km} / \mathrm{h}$. The road labeled "worn DAC" shows a $1.5 \mathrm{~dB}$ higher rolling noise in the frequency range of interest, but also significantly higher emission in the $100-300 \mathrm{~Hz}$ region which can be attributed to stone loss. Higher low frequency vibrations also indicate a possible foundation problem. The road labeled "concrete slabs" consist of $30 \mathrm{~m}$ concrete slabs with intermittent joints. Part of the higher noise emission is due to the wearing of the surface, but the joints may also have an effect. At the same time this road also causes strong low frequency vibrations in cars showing a typical aging effect of this type of pavement. Finally, the highway pavement labeled "concrete" is a continuous concrete on a highway. In accordance to the target speed of $120 \mathrm{~km} / \mathrm{h}$, the amount of low frequency vibrations caused is low confirming the good overall state of the road. Yet, by construction, this type of pavement causes considerable more rolling noise in the frequency range of interest than the SMA.

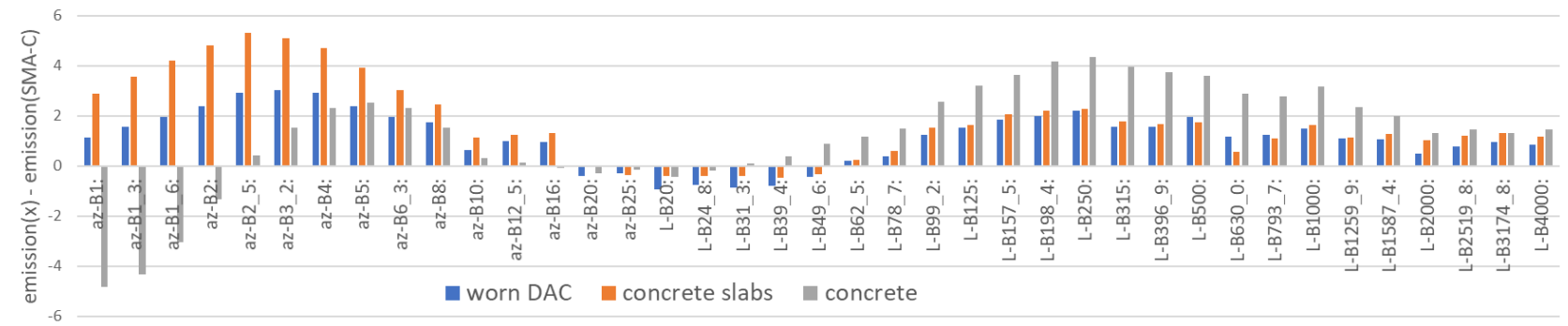

Figure 2 Difference in 1/3 octave band noise emission ( $L-B x x x)$ and vehicle vibration (az-Bxxx) level for three roads near the city of Ghent related to the reference SMA-C surface.

\subsection{Longitudinal analysis}

Figure 3 shows the trend in converged noise levels in the $1000 \mathrm{~Hz}$ octave band for 4 example OSM (open street map) road segments near Ghent, Belgium. Each line in the graph corresponds to a 20m sub-segment. The first three samples are randomly selected and illustrate: (1) noise emission varies along the road and this variation can amount up to $0.5 \mathrm{~dB}$ in some cases for pavements that have been in use for some time; (2) there is a small increasing trend, for which we unfortunately cannot guarantee that it is not caused by a remaining temperature effect (August is usually the hottest month, January the coldest). The fourth example (bottom-right graph on Figure 3) has been selected on purpose to show the effect of road works on a bridge near Ghent. Prior to the intervention, noise emission is very unevenly spread over $20 \mathrm{~m}$ segments and there is some variation over time. The latter may be partly due to lateral displacement of the driving lanes during the road works. March and April data show the effect of the new road surface on the lane that was monitored. Very similar trends are observed for frequencies down to $125 \mathrm{~Hz}$. 

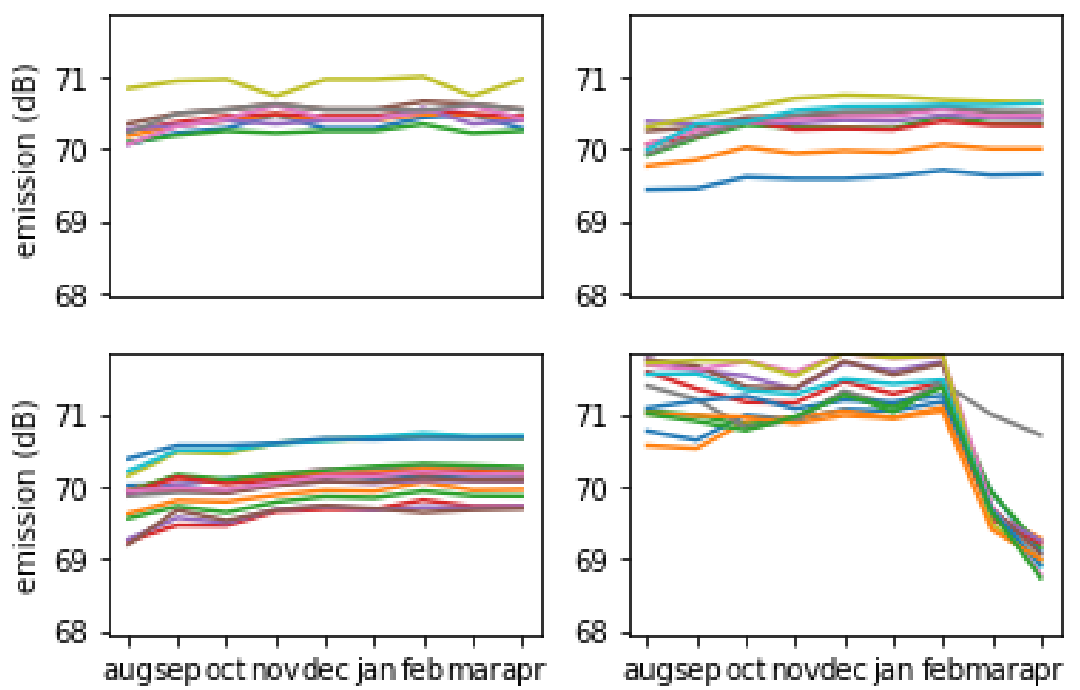

Figure 3 Trend in noise emission at 1000 Hz 1/3 octave band for $20 \mathrm{~m}$ road segments along a OSM road section between August 2020 and April 2021 (each line represents one 20m road segment): upper-left OSM20190830-10377289, upper-right OSM20190830116235506, lower-left OSM20190830-136116628, lower-right OSM20190830-38626857.

\subsection{Spatial analysis}

The DAE model implicitly includes the dependence of noise emission on driving speed. Hence, at first sight, it would be useful to select a fixed target velocity (e.g. $80 \mathrm{~km} / \mathrm{h}$ ) as reference condition. But, this work mainly focusses on urban roads and many pavements that are used on these roads are not suitable for higher speeds so cars will never drive on those at this speed. Thus, it makes more sense to immediately use the specific speed dependence model to express the quality of the road with respect to rolling noise at the typical driving speed at this road. However, there is another caveat. As the DAE model is trained on data that does not contain measurements on these low-speed pavements by cars driving at $80 \mathrm{~km} / \mathrm{h}$ it is not applicable to predict noise emission at these unrealistic target speeds. Hence the 85 percentile of the distribution of driving speeds, v85, is used as a target for the prediction.

Figure 4 gives an example of spatial distribution of the effect of pavement condition on rolling noise emission. The left pane includes the effect of typical driving speed and could be regarded as a measure of impact of the road per vehicle on the sound environment. The right pane shows the deviance of the local road from expected emission based on typical travel speed. It is an indication of the possible effect of a noise management strategy focusing on road surface maintenance.

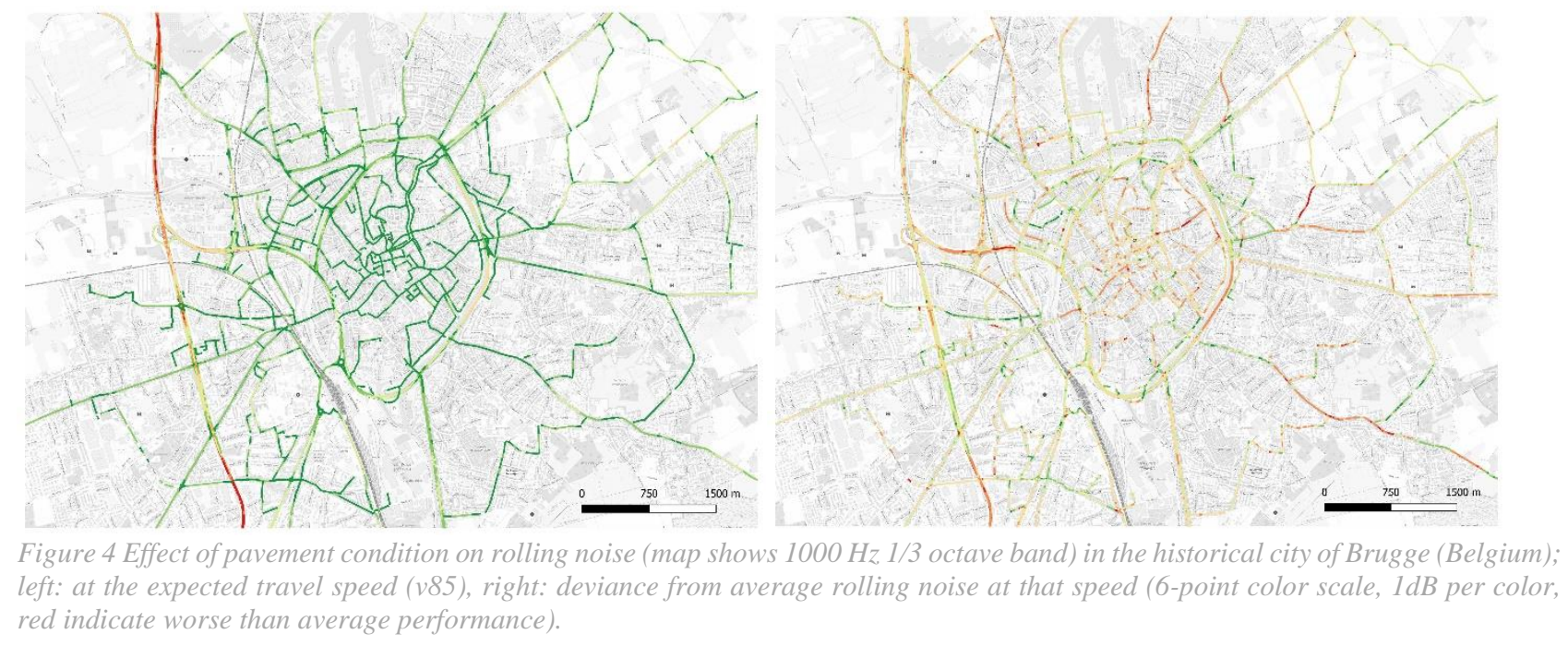




\section{DISCUSSION}

The DAE works well for removing noise from the rolling sound and for calibrating vehicles against each other. This results in an efficient continuous monitoring of the effect of pavement on rolling noise at typical driving speeds and for typical passenger cars. However training of the DAE network proves to be sensitive to parameter settings, network layout, and training dataset. In addition, a device identifier is needed both at the input and the output, so this limits the trained model to a known set of devices. This jeopardizes a fast roll-out in new areas. As training takes quite some computing resources ( 1 week), a strategy needs to be developed to start from a pre-trained model and "finetune" the model to new vehicles. It can further be expected that the calibration capacity of the DAE depends on the overlap in roads traveled by the different devices. The connectivity of vehicles in the current dataset is illustrated in Figure 5. For all of these reasons, a training strategy that avoids the use of unique car identifiers would be beneficial.

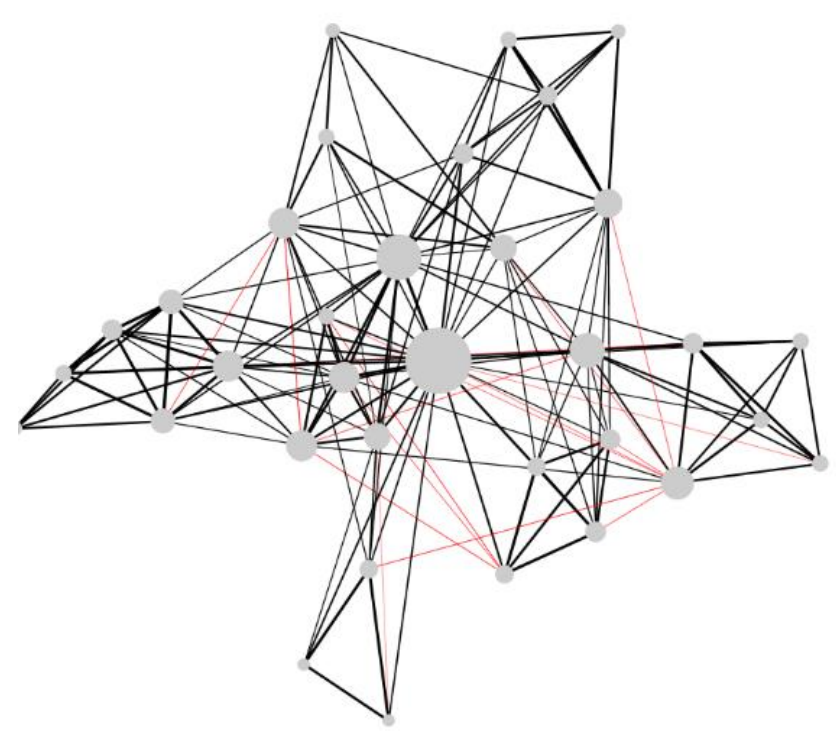

Figure 5 Connectivity of vehicles. Every circle represents a vehicle. Connections between the circles indicate that these vehicles have travelled on the same locations. The stroke width of the edge scales with the logarithm of the amount of pairs of observations which exist between two devices. The circle radius scales with the amount of connections of the device. Some vehicles act as hubs, connecting many other devices. However there are also sparsely connected clusters (for instance the cars in a remote village).

In this publication we focused on classifying and monitoring pavements in relation to the rolling noise produced by the average vehicle. Similar approaches have been proposed by others [6] yet most of this work has been limited to small areas and a much smaller number of vehicles that were well characterized and driven under known conditions. Here, we demonstrate that the approach can be extended to fully opportunistic approaches with a large number of vehicles of various type and make. However, the inverse problem, that is identifying the road surface damage causing the sound is of almost even greater interest. Several methods have been proposed for this [7]. The technique we propose is equally suitable (to be reported elsewhere).

\section{ACKNOWLEDGEMENTS}

This research received funding from the Research Foundation Flanders (FWO-Vlaanderen) [G0D5215N], ERC Runner-up project MAESTRO. In addition, the data has been provided by the ICON project MobiSense [HBC.2017.0155], supported by IMEC and Flanders Innovation \& Entrepreneurship (Vlaio).

\section{REFERENCES}

[1] R. van Loon, C. Tollenaar, and G. van Blokland, Mechanisms of Acoustic Aging of Road Surfaces, in Euronoise 2015 (2020). 
[2] R. Wehr, A. Fuchs, and C. Aichinger, A Combined Approach for Correcting Tyre Hardness and Temperature Influence on Tyre/Road Noise, Applied Acoustics 134, (2018).

[3] T. Vieira, U. Sandberg, and O. Eriksson, A Round Robin Test on the Close-Proximity Method: Comparison of Results from Four Different CPX Trailers Measuring Tyre/Road Noise Properties of 10 Swedish Road Surfaces, Noise Control Engineering Journal 68, (2020).

[4] W. V. Hauwermeiren, J. David, L. Dekoninck, T. de Pessemier, W. Joseph, D. Botteldooren, L. Martens, K. Filipan, and B. de Coensel, Assessing Road Pavement Quality Based on Opportunistic In-Car Sound and Vibration Monitoring, in Proceedings of the 26th International Congress on Sound and Vibration, ICSV 2019 (2019).

[5] W. van Hauwermeiren, K. Filipan, D. Botteldooren, and B. de Coensel, Opportunistic Monitoring of Pavements for Noise Labeling and Mitigation with Machine Learning, Transportation Research Part D: Transport and Environment 90, (2021).

[6] C. Ramos-Romero and C. Asensio, Asphalt-Surface Defects Detection, Based on Tyre/Road Noise Analysis and Geo-Processing, in Proceedings of the International Congress on Acoustics, Vols. 2019-September (2019).

[7] M. R. Ganji, A. Ghelmani, A. Golroo, and H. Sheikhzadeh, A Brief Review on the Application of Sound in Pavement Monitoring and Comparison of Tire/Road Noise Processing Methods for Pavement Macrotexture Assessment, Archives of Computational Methods in Engineering (2020). 